ARMOND, C; OLIVEIRA, VC; GONZALEZ, SDP; OLIVEIRA, FER; SILVA, RM; LEAL, TTB; REIS, AS; SILVA, F. 2016. Desenvolvimento inicial de plantas de abobrinha italiana cultivada com húmus de minhoca. Horticultura Brasileira 34: 439-442. DOI - http://dx.doi.org/10.1590/S0102-05362016003022

\title{
Desenvolvimento inicial de plantas de abobrinha italiana cultivada com húmus de minhoca
}

\author{
Cintia Armond ${ }^{1}$; Vanuze C Oliveira ${ }^{2}$; Sergio DP Gonzalez'; Francisco ER Oliveira $^{\mathbf{1}}$; Rafael M Silva ${ }^{\mathbf{1}}$; \\ Tamara TB Leal'; Ademilde S Reis ${ }^{1}$; Franceli Silva ${ }^{1}$ \\ ${ }^{1}$ Universidade Federal do Recôncavo da Bahia (UFRB), Cruz das Almas-BA, Brasil; franceli.silva@gmail.com; ${ }^{2}$ Universidade Federal de \\ Lavras (UFLA), Lavras-MG, Brasil; vanuze.costa@gmail.com
}

\section{RESUMO}

A abobrinha é uma das dez hortaliças mais cultivadas no Brasil. Possui alto valor nutricional e, por isso é utilizada como ingrediente principal na produção de tortas, doces e ração animal. Apesar da importância econômica e nutricional, poucos são os trabalhos envolvendo a adubação e propagação da cultura. Neste contexto, objetivou-se avaliar o efeito do húmus de minhoca no desenvolvimento de plantas jovens de abobrinha. O experimento foi realizado durante os meses de novembro de 2012 a janeiro de 2013, em casa de vegetação. $O$ delineamento estatístico foi inteiramente casualizado, com seis tratamentos $(0,100,200,300,400$ e $1000 \mathrm{~g}$ de húmus de minhoca por vaso de $1,5 \mathrm{~L}$ ) e quatro repetições. As plantas cultivadas em $1000 \mathrm{~g}$ de húmus de minhoca apresentaram maiores teores de massa seca da folha, caule e raiz. Pela análise de regressão, observou-se que a dosagem de $750 \mathrm{~g}$ foi a que permitiu os máximos valores de razão de área foliar e razão de peso foliar. Identificou-se relação positiva entre a proporção de húmus e a massa seca de folhas+caule, massa seca da raiz e a massa seca total. Os teores de clorofila $a$ e $b$ foram maiores em doses de 575 e 644 g, respectivamente. Assim sendo, o uso de húmus de minhoca favorece o desenvolvimento fisiológico de plantas jovens de abobrinha.

Palavras-chave: Cucurbita pepo, adubação orgânica, teores de clorofila, massa seca de plantas, desenvolvimento fisiológico.

\begin{abstract}
Initial development of Italian Zucchini plants cultivated with worm humus

Zucchini is one of the ten most important vegetable crops grown in Brazil. It has a high nutritional value, and is used as main ingredient of cakes, candies and animal food. Despite the economical and nutritional importance, few researches are available about its propagation and fertilization. The main objective of this work was to evaluate the effect of the earthworm humus on the development of young zucchini plants. The experiment was carried out in a greenhouse from November (2012) to January (2013) in a complete randomized design, with six doses $(0,100,200,300,400$ and $1000 \mathrm{~g}$ of earthworm humus per a 1,5 L pot) and four replications. The plants grown in $1000 \mathrm{~g}$ had higher levels of dry mass of leaf, stem and root. Regression analysis showed that the dosage of $750 \mathrm{~g}$ allowed the maximum values of leaf area ratio and leaf weight ratio variables. A positive relationship was identified between the proportion of humus and the dry mass of leaves + stem, root dry mass and total dry mass. $a$ and $b$ chlorophyll levels were higher at doses of 575 and $644 \mathrm{~g}$, respectively. Therefore, the use of earthworm humus favors the physiological development of young zucchini plants.
\end{abstract}

Keywords: Cucurbita pepo, organic fertilization, chlorophyll content, dry weight of plants, physiological development.

(Recebido para publicação em 6 de agosto de 2015; aceito em 20 de abril de 2016)

(Received on August 6, 2015; accepted on April 20, 2016)

A abobrinha (Cucurbita pepo), pertencente à família Cucurbitaceae, é conhecida no Brasil como abóbora de moita, abobrinha italiana, caserta ou abobrinha de tronco (Filgueira, 2008). Esta espécie está entre as dez hortaliças de maior valor econômico no País (Carpes et al., 2008).

Apesar da importância econômica da abobrinha, poucos estudos têm sido realizados em relação à sua propagação e fertilização, incluindo a adubação orgânica. No Brasil, existe a necessidade de mais estudos quanto às dosagens de fertilizantes utilizados nas culturas, adequados às diferentes cultivares, regiões e épocas de plantio de abobrinhas (Silva et al., 2011). O fato justifica-se por, almejando maior produtividade, o olericultor aplicar doses elevadas de elementos minerais, resultando, na maioria das vezes, em distúrbios nutricionais às plantas, além de aumentar o custo de produção (Resende et al., 2005). O consumo de luxo, proporcionado por doses elevadas de determinado elemento aplicado nas plantas, não resulta em aumento na produção.

O uso de material orgânico na adubação tem contribuído para o aumento da produtividade de diversas culturas agrícolas, por favorecer a fertilidade do solo (Freitas et al., 2012). Além disso, com o uso de materiais orgânicos, quando ricos em nitrogênio, por exemplo, é possível proporcionar o aumento na absorção de diversos nutrientes, como P, K, Ca, Mg (Souza et al., 2015). A adubação orgânica tem se mostrado eficiente no crescimento e na produção de várias culturas (Corrêa et al., 2012; Souza et al., 2015).

Quando se trata de espécies de ciclo curto, como a abobrinha, o uso de húmus de minhoca apresenta-se como 
alternativa para proporcionar maior desenvolvimento de tais culturas, já que este adubo realiza a disponibilização mais rápida de nutrientes para a planta.

Além disso, é importante considerar que a produção de mudas é uma das etapas mais importantes na produção das hortaliças, já que a qualidade das mudas determinará o desempenho produtivo destas plantas (Sediyama et al., 2014). Neste sentido, Santos et al. (2010), ao utilizarem substratos constituídos por vermicomposto, mostraram que o uso destes substratos contribuiu com o aumento no índice SPAD em mudas de pimentão, sendo este índice relacionado aos teores de nitrogênio nas plantas e, consequentemente, nos teores de clorofila.

Neste contexto, objetivou-se avaliar o comportamento fisiológico de plantas jovens de abobrinha, quando submetidas a doses de húmus de minhoca.

\section{MATERIAL E MÉTODOS}

O experimento foi conduzido na casa de vegetação de nutrição mineral de plantas da Universidade Federal do Recôncavo da Bahia, no Município de Cruz das Almas-BA.

As plantas de abobrinha (cultivar Caserta) foram obtidas através da semeadura de quatro sementes em recipientes plásticos com capacidade para 1,5 L, contendo como substrato areia lavada além do húmus de minhoca nas doses de: 0, 100, 200, 300, 400 e 1000 g. A composição química do húmus de minhoca foi $\mathrm{pH}=7,10 ; \mathrm{Al}$ trocável $=0,0$ $\mathrm{cmol}_{\mathrm{c}} / \mathrm{dm}^{3} ; \mathrm{Ca}+\mathrm{Mg}=15,6 \mathrm{cmol}_{\mathrm{c}} / \mathrm{dm}^{3} ; \mathrm{P}=$ $90,0 \mathrm{mg} / \mathrm{dm}^{3} ; \mathrm{K}=780,0 \mathrm{mg} / \mathrm{dm}^{3}$; matéria orgânica $=42,30 \%$. Foi adotado o delineamento inteiramente casualizado com quatro repetições, sendo que cada vaso constituiu uma parcela experimental.

Dez dias após a emergência, foi realizado o desbaste, ficando apenas uma planta por vaso. Em seguida, foram realizadas as avaliações das variáveis clorofila $a$ e $b$ com o clorofilômetro (Clorofilog CFL1030), em folhas medianas das plantas, sendo a medição sempre realizada na porção proximal da folha; e também foram medidas a altura e o diâmetro do caule das plantas. Tal processo avaliativo foi realizado a cada dois dias durantes duas semanas, totalizando sete avaliações.

Na última avaliação as plantas foram colhidas e separadas em folhas, caules e raízes. Foi determinada a área foliar com integrador de área foliar (AREA METER-AM 300). A parte aérea e as raízes foram acondicionadas em sacos de papel e transferidas para estufa de circulação forçada de ar, a $70^{\circ} \mathrm{C}$, até atingir peso constante. Obtendo-se a massa seca e área foliar, foi determinada a Razão de Área Foliar (RAF) e a Razão de Peso Foliar (RPF). Para tanto, utilizaram-se as seguintes equações:

$$
\begin{aligned}
& \mathrm{RAF}=\mathrm{L} / \mathrm{W}(1) ; \\
& \mathrm{RPF}=\mathrm{RAF} / \mathrm{AFE}(2)
\end{aligned}
$$

Em que, RAF é a razão de área foliar; L é a área foliar; W consiste na massa seca total da planta; RPF é a razão de peso foliar e AFE é a área foliar específica. AAFE relaciona a superfície com a massa da matéria seca da própria folha e é expressa em $\mathrm{cm}^{2} / \mathrm{g}$, conforme Peixoto et al. (2011).

Os dados foram submetidos à análise de variância e análise de regressão, utilizando o programa estatístico SAS 9.0.

\section{RESULTADOS E DISCUSSÃO}

Houve efeito significativo da adubação com húmus de minhoca na produção de massa seca da abobrinha. Observou-se que a massa seca total, de folhas e caule e de raízes, desenvolveu tendência linear (Tabela 1). Isso significa que, maior dose de húmus de minhoca resulta em maior incremento na produção de massa seca em plantas de abobrinha.

Maior massa seca total estimada em $5,0 \mathrm{~g}$ foi obtida na maior dose de húmus de minhoca. O aumento da massa seca nas diferentes partes da planta relacionada com o aumento de dosagens de húmus, também foi encontrado por Góes et al. (2011).

É necessário conhecer os níveis adequados de nutrientes em cada espécie para reduzir custos com adubação, aumentar a produção e evitar efeitos fitotóxicos. A quantidade de nutrientes exportados pela cultura é um componente importante na perda de nutrientes do solo, devendo ser considerado na

Tabela 1. Parâmetros de crescimento e teores de clorofila em plantas jovens de abobrinha submetidas a doses de húmus de minhoca (growth parameters and chlorophyll levels in young zucchini plants to worm humus doses). Cruz das Almas, UFRB, 2013.

\begin{tabular}{lcccc}
\hline Variáveis & Equação & $\mathbf{R}^{2}$ & $\begin{array}{c}\text { Dose estimada } \\
(\mathbf{g})\end{array}$ & $\begin{array}{c}\text { Maior valor para as } \\
\text { variáveis avaliadas }\end{array}$ \\
\hline $\mathrm{RPF}(\mathrm{g} / \mathrm{g})$ & $\hat{\mathrm{Y}}^{* *}=0,244+9.10^{-4} \mathrm{X}-6 \times 10^{-7} \mathrm{X}^{2}$ & 66,98 & 750 & 0,58 \\
$\mathrm{RAF}\left(\mathrm{cm}^{2} / \mathrm{g}\right)$ & $\hat{\mathrm{Y}}^{*}=0,008+10^{-5} \mathrm{X}-7 \times 10^{-9} \mathrm{X}^{2}$ & 61,55 & 555 & 0,012 \\
$\mathrm{MSPA}(\mathrm{g})$ & $\hat{\mathrm{Y}}^{* *}=0,1861+0,0035 \mathrm{X}$ & 98,83 & 1000 & 3,68 \\
$\mathrm{MSR}(\mathrm{g})$ & $\hat{\mathrm{Y}}^{* *}=0,1007+0,0012 \mathrm{X}$ & 79,62 & 1000 & 1,30 \\
$\mathrm{MST}(\mathrm{g})$ & $\hat{\mathrm{Y}}^{* *}=0,2868+0,0047 \mathrm{X}$ & 97,01 & 1000 & 5,00 \\
$\mathrm{CLO} a(\mathrm{mg} / \mathrm{g})$ & $\hat{\mathrm{Y}}^{* *}=19,96+0,023 \mathrm{X}-2 \times 10^{-5} \mathrm{X}^{2}$ & 80,42 & 575 & 26,56 \\
$\mathrm{CLO} b(\mathrm{mg} / \mathrm{g})$ & $\hat{\mathrm{Y}}^{* *}=6,343+0,0116 \mathrm{X}-9 \times 10^{-6} \mathrm{X}^{2}$ & 86,91 & 644 & 13,80 \\
\hline
\end{tabular}

$\mathrm{RPF}=$ razão de peso foliar (leaf weight ratio); $\mathrm{RAF}=$ razão de área foliar (leaf area ratio); MSPA= massa seca da parte aérea (dry weight of aboveground part); MSR = massa seca da raiz (root dry mass); MST= massa seca total (total dry matter); CLO $a=$ clorofila $a$ (chlorophyll $a$ ); CLO $b=$ clorofila $b$ (chlorophyll $b$ ). *** significativo ao nível de 5 e $1 \%$ de probabilidade, respectivamente (significant at 5 and $1 \%$ probability, respectively). 
hora de definir o manejo da adubação para reposição de nutrientes (Santos et al., 2012).

Das variáveis relacionadas ao crescimento de plantas, apenas a razão de área foliar (RAF) e a razão de peso foliar (RPF) foram influenciadas significativamente pela dose de húmus de minhoca, ambas apresentando resposta quadrática às doses de matéria orgânica utilizada (Tabela 1). A diferença percentual entre a maior razão de área foliar $(0,012$ $\mathrm{cm}^{2} / \mathrm{g}$ ) e a menor razão de área foliar $\left(0,0084 \mathrm{~cm}^{2} / \mathrm{g}\right)$ foi equivalente a $70 \%$, sendo este maior valor estimado na dose de $555 \mathrm{~g}$ de húmus de minhoca.

De acordo com Peixoto et al. (2011), a RAF é a relação de quanto de área foliar é necessário para produzir um grama de matéria seca total. A RAF pode ser influenciada por diversos fatores além da adubação, como a idade das plantas (Araújo Junior et al., 2012).

Ao trabalhar com adubação orgânica, sendo os adubos utilizados esterco bovino e o esterco de aves, Corrêa et al. (2010) obtiveram maiores valores de RAF em plantas de orégano, quando adubadas com esterco bovino. Os autores relacionaram o aumento dos valores de razão de área foliar à maior concentração de nitrogênio, já que este nutriente é considerado um dos nutrientes que mais influencia a produtividade, por estar ligado à fotossíntese e ao crescimento das plantas (Yin et al., 2003).

Para a RPF, estimada pela equação da Tabela 1, tem-se que a dose de $750 \mathrm{~g} /$ $\mathrm{kg}$ de húmus de minhoca proporcionou a maior razão de peso foliar, sendo o seu maior valor $0,58 \mathrm{~g} / \mathrm{g}$. A diferença percentual entre o maior $(0,58 \mathrm{~g} / \mathrm{g})$ e o menor valor $(0,16 \mathrm{~g} / \mathrm{g})$ para a RPF foi de $72 \%$.

A avaliação desses parâmetros de crescimento vegetal é importante segundo Peixoto et al. (2011), devido a RPF expressar a quantidade de massa não transferida das folhas para outras partes da planta, especialmente em plantas cujo interesse econômico está nas folhas, como as hortaliças folhosas. Por outro lado a RAF serve como parâmetro apropriado para as avaliações de efeitos climáticos e do manejo de vegetais.

Utilizando adubação orgânica na cultura do Origanum vulgare, Corrêa et al. (2010) constataram que para RPF não houve efeito significativo das doses dos adubos, o que implica dizer que a adubação orgânica não contribuiu com a exportação de material produzido nas folhas para as outras partes da planta como caule e raiz. Também, trabalhando com adubação orgânica, Janegitz et al. (2011) verificaram que a utilização do adubo orgânico afetou significativamente as variáveis relacionadas ao crescimento de plantas na cultura da mamoneira.

O húmus serve como fonte de energia e nutrientes para o desenvolvimento de muitos grupos de organismos, principalmente microrganismos e, como resultado de sua decomposição, há a liberação contínua de $\mathrm{CO}_{2}, \mathrm{NH}^{4+}$, íons de $\mathrm{P}, \mathrm{S}$, e micronutrientes, que são de grande importância para o ciclo dos vegetais, o que está diretamente relacionado à fotossíntese $\mathrm{e}$, consequentemente à formação de frutos.

O maior teor de clorofila $a$, estimado em $26,56 \mathrm{mg} / \mathrm{g}$ foi obtido na dosagem 575 g da matéria orgânica; para o teor de clorofila $b$, a dose de 644 g proporcionou o maior valor, estimado em 13,80 $\mathrm{mg} / \mathrm{g}$ (Tabela 1). Os maiores valores de clorofila podem estar relacionados às doses crescentes de adubação orgânica, até o ponto de máximo da curva que, podem ser explicados pela maior disponibilidade de nutrientes como nitrogênio e magnésio, considerando que tais elementos são constituintes da molécula de clorofila. Por outro lado, o decréscimo no teor de clorofila, a partir do ponto de máximo da curva, pode ser atribuído ao excesso de nutrientes no substrato, causando redução do pigmento (Ferreira et al., 2012). Ou seja, no momento em que ocorre aumento da disponibilidade de nutrientes, atinge-se um ponto em que uma adição continuada dos mesmos não contribui com o aumento no crescimento ou produtividade, resultando em aumentos na concentração dos nutrientes nos tecidos, sem, no entanto, haver a assimilação destes por parte dos vegetais.

A disponibilidade de nutrientes como o nitrogênio e o magnésio pode explicar os maiores valores de clorofila, obtidos em função de doses crescentes de adubação orgânica. A concentração de clorofila $a$ e $b$ na planta tem correla- ção positiva com o teor de nitrogênio na folha, influenciando a taxa fotossintética das plantas, sendo que a concentração de nitrogênio pode ser favorecida pela aplicação de produtos de origem orgânica (Zhao et al., 2005; Ruttanaprasert et al., 2012). É possível supor que ocorreu a assimilação de nutrientes pelas plantas e que a adição de húmus de minhoca a substratos inertes como a areia supriu as necessidades nutricionais das fases iniciais das plantas de abobrinha.

Considerando os resultados obtidos, pode-se concluir que o uso de húmus de minhoca em doses superiores a $500 \mathrm{~g}$ é favorável ao desenvolvimento fisiológico de plantas jovens de abobrinha.

\section{AGRADECIMENTOS}

Ao CNPq, à Capes e à FAPESB pelo apoio financeiro e a concessão de bolsas de estudo.

\section{REFERÊNCIAS}

ARAÚJO JUNIOR, BB; SILVA, PSL; MORAIS, PLD; DOMBROSKI, JLD; OLIVEIRA, VR. 2012. Crescimento do milho com controle de plantas daninhas via consorciação com gliricídia. Planta Daninha 30: 757-766.

CARPES, RH; LÚCIO, AD; STORCK, L; LOPES, SJ; ZANARDO, B; PALUDO, AL. 2008. Ausência de frutos colhidos e suas interferências na variabilidade da fitomassa de frutos de abobrinha italiana cultivada em diferentes sistemas de irrigação. Revista Ceres 55: 590-595.

CORREAA, RM; PINTO, JEBP; REIS, ES; COSTA, LCB; ALVES, PB; NICULAN, ES; BRANT, RS. 2010. Adubação orgânica na produção de biomassa de plantas, teor e qualidade de óleo essencial de orégano (Origanum vulgare L.) em cultivo protegido. Revista Brasileira de Plantas Medicinais 12: 80-89.

CORREAA, RM; PINTO, JEB; REIS, ES; MOREIRA, CM. 2012. Crescimento de plantas, teor e qualidade de óleo essencial de folhas de orégano sob malhas coloridas. Global Science and Technology 5: 11-22.

FERREIRA, TA; SILVA, EHC; RIBEIRO, MMC; CHAVES, PPN; NASCIMENTO, IR. 2012. Acúmulo de clorofila e produção de biomassa em hortelã-verde sob diferentes níveis de adubação orgânica. Revista Verde de Agroecologia e Desenvolvimento Sustentável 7: 41-45.

FILGUEIRA, FAR. 2008. Novo Manual de Olericultura. 3.ed. Viçosa: Editora UFV, 421p. FREITAS, MSC; ARAÚJO, CAS; SILVA, DJ. 
2012. Decomposição e liberação de nutrientes de estercos em função da profundidade e do tempo de incorporação. Revista Semiárido De Visu 2: 150-161.

GÓES, GB; DANTAS, DJ; ARAÚJO, WBM; MELO, IGC; MENDONÇA, V. 2011. Utilização de húmus de minhoca como substrato na produção de mudas de tamarindeiro. Revista Verde 6: 125-131.

JANEGITZ, MC; HERMANN, ER; MATOSO, A. 2011. Adubos orgânicos no desenvolvimento inicial de mamoneira em solo corrigido com Calcário. Cascavel 4: 73-82.

PEIXOTO, CP; CRUZ, TV; PEIXOTO, MFSP. 2011. Análise quantitativa do crescimento de plantas: Conceitos e prática. Enciclopédia Biosfera, Centro Cientifico Conhecer 7: 51-76.

RESENDE, GM; ALVARENGA, MAR; YURI, JE; MOTA, JH; SOUZA, RJ; RODRIGUES, JÚNIOR, JC. 2005. Produtividade e qualidade pós-colheita da alface americana em função de doses de nitrogênio e molibdênio. Horticultura
Brasileira 23: 976-981.

RUTTANAPRASERT, R; JOGLOY, S; VORASOOTN, KT; KANWAR, RS; HOLBROOK, CC. 2012. Relationship between chlorophyll density and spad chlorophyll meter reading for Jerusalem Artichoke (Helianthus tuberosus L.). Journal of Breeding and Genetics 44: 149-162.

SANTOS, MR; SEDIYAMA, MAN; MOREIRA, MA; MEGGUER, CA; VIDIGAL, SM. 2012. Rendimento, qualidade e absorção de nutrientes pelos frutos de abóbora em função de doses de biofertilizante. Horticultura Brasileira 30: 160-167.

SANTOS, MR, SEDIYAMA, MAN, SALGADO, LT, VIDIGAL, SM; REIGADO, FR. 2010. Produção de mudas de pimentão em substratos à base de vermicomposto. Bioscience Journal 26: 572-578.

SEDIYAMA, MAN; SANTOS, IC; LIMA, PC. 2014. Cultivo de hortaliças no sistema orgânico. Revista Ceres 61: 829-837.
SILVA, LV; OLIVEIRA, GQ; SILVA, MG; NAGEL, PL; MACHADO, MMV. 2011. Doses de nitrogênio em cobertura em duas cultivares de abobrinha no município de Aquidauana-MS. Revista Brasileira de Ciências Agrárias 6: 447-451.

SOUZA, JL; GUIMARÃES, GP; FAVARATO, LF. 2015. Desenvolvimento de hortaliças e atributos do solo com adubação verde e compostos orgânicos sob níveis de $\mathrm{N}$. Horticultura Brasileira 33: 019-026.

YIN, X; LANTINGA, A; SCHAPENDONK, HCM; ZHONG, X. 2003. Some quantitative relationships between leaf area index and canopy nitrogen content and distribution. Annals of Botany 91: 893-903.

ZHAO, D; REDDY, R; KAKANI, GV; READ, JJ. 2005. Selection of optimum reflectance ratios for estimating leaf nitrogen and chlorophyll concentrations of field-grown cotton. Agronomy Journal 98: 89-98. 